\title{
Self Injurious Behavior in Temporal Lobe Epilepsy
}

\author{
Shakya DR, ' Shyangwa PM, ${ }^{1}$ Pandey AK, ${ }^{1}$ Subedi $S,{ }^{1}$ Yadav $\mathrm{S}^{1}$ \\ 'Department of Psychiatry, B P Koirala Institute of Health Sciences, Dharan.
}

\section{ABSTRACT}

Self-injurious behavior (SIB) or self-mutilating behavior (SMB) is rare but can occur in temporal lobe epilepsy. Such a behavior during seizures is not usually recalled by patient. Here is a case with self mutilating behavior in left temporal lobe epilepsy, presented because of its rare manifestation and diagnostic dilemma.

A 19 year old unmarried Muslim student presented to emergency with SMB, guilty rumination and a persecutory delusion. The patient was intermittently confused about place and time. In subsequent assessments, he was found harboring death wishes and suicidal ideation. He transiently had auditory hallucination and thought broadcasting. He episodically tried to harm himself by severely biting only his left ring finger. It was difficult to influence him during such episodes. EEG revealed left temporal lobe seizure. Diagnosis of 'Epilepsy and Organic Psychosis' was made. The patient responded well to Antiepileptic and Antipsychotic medications.

Keywords: seizure, self injurious behavior, suicide, temporal epilepsy

\section{INTRODUCTION}

Though rare, self injurious or mutilating behavior (SIB/ SMB) is seen in seizure cases. ${ }^{1,2}$ Suicide behaviors are more common in seizure cases than in general population. ${ }^{3-6}$ The presented case displayed both SIB and suicidal ideation (in the form of forced thinking along with guilty rumination) which the patient did not recall after the control of seizure with antiepileptic and antipsychotic medication.

\section{CASE REPORT}

A 19 year unmarried, Muslim, male student; also a social activist from a village, presented to emergency room with chief complaints of guilty rumination and repeatedly biting his left ring finger for eight days. The patient abruptly had the guilt of eating non-halal meat cooked by Hindus, which he did a few days before at a social activity. He, at times felt as if he was drawn into the worry about being outcasted in his society because of eating it. This information was not known to his society people and according to the family members, there was little basis for his worry.

After a difficult night of sleep, he began to claim that people were trying to kill him. He appeared fearful and at times irritable. He sometimes expressed, "Hindus can't kill me" and suddenly would bite his left ring finger causing severe bleeding. During such episodes which lasted for 20-40 minutes, he could not be

Correspondence:

Dr. Dhana Ratna Shakya

Department of Psychiatry

B P Koirala Institute of Health Sciences, Dharan.

Email: drdhanashakya@yahoo.com

Phone: 025525555 Ext. 5347 
Shakya et al. Self Injurious Behavior in Temporal Lobe Epilepsy

influenced and he manifested restlessness, sweating, and disorientation to time and place. He had to be forcefully prevented from biting. During such episodes, two incisor teeth were accidentally uprooted when others tried to pull his hand out of his mouth.

There was no similar or other neuro-psychiatric or medical illness, nor any history of head injury or CNS infection in the past. His cousin and aunt had seizures; and, maternal uncle (third degree relative) had suffered an illness suggestive of psychosis. He does not take any psycho-active substance, and was well adjusted pre-morbidly. The senior members of family were more religious and regularly visit Mosque for the prayers.

There was no abnormal finding in general physical survey and systemic examination, including CNS, except local findings- multiple lacerations in left ring finger and missing two lower incisor teeth. He was restless and fearful; had to be brought to examination room, rapport was not established because of poor communication and was disoriented in place and time on some examinations. Other time, he was relatively quiet. However, he expressed guilt about eating non-halal meat, and had delusion of being killed and outcasted by his society. In distress, he appeared to harbor suicidal ideation with no elaborate plan. He thought that his ideas were known to others automatically without telling them. He said that intermittently and transiently he heard people talking to each other about him when he did not see anyone around. During ward course, he had frequent episodes of biting of his finger.

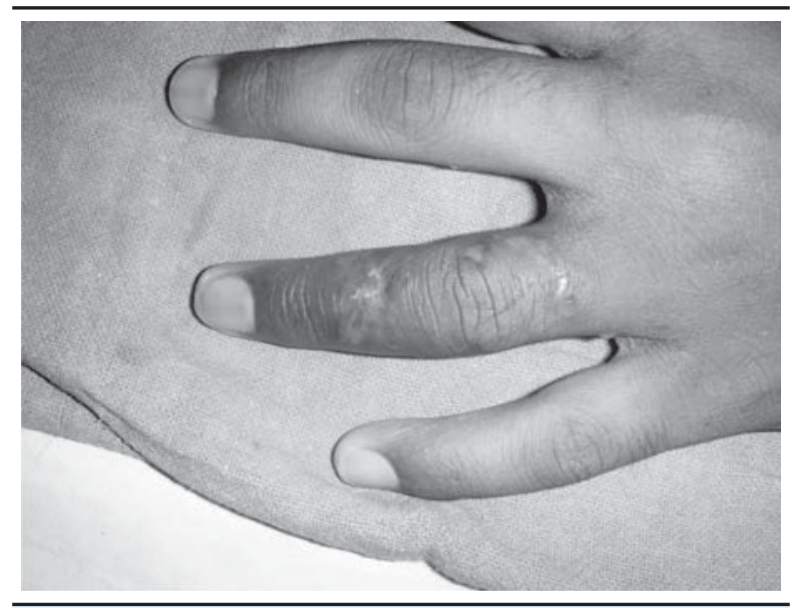

Figure 1. Left ring finger the patient used to bite episodically

Initially, Olanzapine and Lorazepam were administered. EEG in waking state revealed left temporal lobe abnormality. CT scan of head was normal. Electrolytes, blood counts, blood sugar and urine routine were normal. Diagnosis of 'Organic psychotic disorder- delusional in Seizure disorder- Temporal Lobe Epilepsy' was made.
Parenteral, followed by oral Sodium-valproate was given and patient improved steadily and was discharged from the hospital. Upon improvement, he said he did not remember biting the finger and being fearful.

\section{DISCUSSION}

Self-injurious behavior (SIB), self-mutilation (SMB), selfinflicted violence/ wound or self-harm is a 'behavior in which persons hurt or harm themselves without the motive of suicide' ${ }^{7}$ Self-mutilation is inflicted most commonly by cutting oneself with a sharp object; and by burning with cigarettes, inserting objects under the skin, head banging, nail/ finger biting, hair pulling, etc. Though most of SIB/ SMB are deliberate, intentional or voluntary as in impulsive, attention seeking, relief directed, because of distress or personality problems; there are also other conditions where a person might end up with involuntary or non deliberate SIB/ SMB, as in organic conditions, e.g. seizures. ${ }^{1-3}$

Similarly, suicide behaviors are also more common among seizure cases than they are in general people. ${ }^{3}$ $6,8,9$ Suicide phenomena are a spectrum of deliberate self harm behaviors; with death wishes, suicidal-ideation, plan, attempt and completed, with intention to end owns life. ${ }^{8}$ Seizure patients may show suicidal behavior during different phases of seizure or in psychiatric conditions associated or co-morbid with seizure..$^{8,9}$

Seizure disorder or epilepsy is one of the most common neuro-psychiatric disorders affecting approximately $1 \%$ of population. ${ }^{3}$ It ranks the sixth position in the list of the most important neuro-psychiatric causes in terms of chronic disability. ${ }^{10}$ Seizure is defined as 'an abnormal movement or behavior caused by unusual electrical activity in the brain' ${ }^{3,4}$ Epilepsy is a group of related disorders characterized by a tendency for recurrent seizures, operationally defined as idiopathic seizure with two or more episodes. ${ }^{3,4}$

Seizure and epilepsy are associated with a wide range of psychiatric disorders in higher proportion than general population (20-60\%). ${ }^{3-6}$ A study carried out in seizure cases referred to Department of Psychiatry, BP Koirala Institute of Health Sciences for some psychiatric problems showed that $45 \%$ fulfilled ICD-10 criteria for one or other mental and behavioral disorders, and the rest had one or other clinically significant symptomatology in 'Brief Psychiatric Rating Scale'.11 These changes may be chronic and present with history of seizures, or episodic and directly related with different phases of seizure activities- aura, peri-ictal, inter-ictal or postictal periods. Frequently, it is difficult to distinguish independent or secondary to seizure activity but it has been consistently seen that mood, anxiety and other neurotic spectrum, psychotic disorders, personality 
Shakya et al. Self Injurious Behavior in Temporal Lobe Epilepsy

and cognitive changes, and suicide and self injurious behaviors are seen in these cases. ${ }^{3,12}$ The study in BPKIHS also replicated the same finding. ${ }^{11}$

Among these psychiatric associations, psychotic disorders are also seen (5-9 times that of population). ${ }^{3-6}$ Psychotic symptoms- delusions, hallucinations, disorganized behavior or speech may be seen in postictal or ictal or inter-ictal phases. ${ }^{13}$ Literature, accordingly mainly reveals the types- chronic ictal (secondary/ symptomatic), post-ictal or inter-ictal psychosis. These patients usually do not have family history of psychotic illness.

The features in these psychotic seizure patients are characterized basically by schizophrenic (delusion: grandeous, religious, paranoia), affective (elevated $/ \mathrm{mood}$ swings, agitation) and confusional elements (impulsive behavior, perplexity, disorientation). Though some 'Schneiderian First rank symptoms' might be present and features may not be very different, these psychotic symptoms are found less severe and less persistent than in schizophrenia. The content of the cognition/ thought (both ideational and perceptual) in psychotic seizure patients is limited to one theme or so or related to very similar theme (cognitive stereotypy). In our case also, patient had guilty rumination regarding eating non halal meat, forced thinking about being persecuted by his relatives and Hindus, then death wishes and suicidal ideation, and even self mutilating behavior intermittently (which patient could not remember after the control of seizure). These all cognitive contents are confined to a single theme to which patient was engrossed during seizure phenomenon. The patient was distressed, agitated (affective element) and during such periods, he could not be influenced, did not recognize the place and time, appeared confused (confusional element). After the control of seizure, the patient was surprised when he was asked 'Why did he use to bite his left ring finger?' He appeared worried about his behavior and rather asked 'why had I those problems which I do not remember now?'

Post-ictal variety of psychosis usually develops after lucid interval of 1-3 days of exacerbations of seizures and lasts 16- 432 hours, and spontaneous recovery is expected with seizure control. ${ }^{4}$ Inter-ictal one usually develop after 10-15 years of poorly controlled CPS and auras, and psychosis alternate with seizure..$^{3,4}$

All; ictal, post-ictal or inter-ictal psychosis are related to seizure with partial- both simple or complex, with focus mainly in temporal lobe, mainly left and mes/dio-basal part as seen in most functional studies. ${ }^{3,13}$ EEG shows an increased focal discharge activity, as in this case. The cause for the development of psychiatric symptoms in seizures is manifold- in many cases unknown; in others the same patho-physiology is responsible for both epileptiform activities and behavioral changes; absence of function, neuro-endocrine and transmitter changes and psycho-dynamic factors e.g. helplessness, dependency, decreased self esteem, stigma attached or stressful life events (possibly as in this case). ${ }^{3-}$ 4,12 Theoretically also, temporal lobe is implicated for psychotic symptoms like delusions, hallucinations, besides disturbances in other functions like memory, emotion, language comprehension, audition and sensory prosody. ${ }^{4}$ Many times, the involvement of frontal lobe shows the features like- behavioral changes, aggression, lack of personal care/ appearance, sexual dis-inhibition.

Among different classes of epileptic seizures, complex partial seizure is most commonly associated with ictal psychic symptomatologies. ${ }^{3-5}$ Clinical cues of CPS may be- consistent pattern of episodes with similar and typical subjective/ affective alterations, and other symptoms; clinical features like automatism, confusion, impaired memory, and post-ictal lethargy, depression; symptoms being episodic with short duration of minutes. It may be difficult to diagnose even with nasopharyngeal EEG, and 'Prolactin estimation' may help.

Detailed history, from witness is the mainstay of diagnosis. EEG is helpful in confirmation or in making differential diagnoses. CT/ MRI may help to rule out specific causes. Though 'video- EEG' is the gold standard of diagnostic method, it is not practically possible because of unavailability in our context.

If psychopathology is seizure related, treat the seizure. Control of seizure is mandatory in the management of psychiatric symptoms, including psychosis. Choice of anti-epileptic drugs (AEDs) depends on type of seizures, adverse effects and drug interactions. Mono-therapy is sought as far as possible. Adequate dosing is the principle, and serum level estimation is helpful. AEDs with psychotropic effects, e.g. Sodium-valproate and Carbamazepine may be considered.

It is like that of other psychosis. But, Antipsychotic drug, which does not significantly decrease seizure threshold would be the choice, e.g. Haloperidol, Risperidon, Quetiapine are found to be seizure friendly; and Clozapine, Chlorpromazine are the worst. When Clozapine has to be used, it should be slowly titrated, be monitored and the dose should not exceed $600 \mathrm{mg} /$ day to prevent seizures. ${ }^{4}$ Adding or withdrawing Carbamazepine to or from antipsychotic needs careful consideration, as Carbamazepine can lower antipsychotic serum levels. 'Clozapine + Carbamazepine' combination should be avoided because of higher chance of bone marrow suppression. ${ }^{4}$ 
When patients present with SIB/ SMB in seizure, we should provide measures for prevention of injury and mutilation, and control behavior with antipsychotic/ physical restraints and closely monitor, besides controlling seizures.

\section{ACKNOWLEDGEIMENTS}

Connell S, University of Washington; Shakya R and Adhikari BR, Department of Psychiatry and Thapa LJ, Department of Internal medicine, BPKIHS, Nepal.

\section{REFERENCES}

1. Critchley HD, Simmons A, Daly EM, Russell A, van Amelsvoort T, Robertson DM, et al. Prefrontal and medial temporal correlates of repetitive violence to self and others. Biological Psychiatry. 2000;47(10):928-34.

2. Yuji I, Tsutomu M. Traumatic luxation of the testis due to self-mutilation: A Case Report. Japanese Journal of Urology. 2006;97(1):57-9.

3. Raghuthaman G, Jacob KS, Ranjith G. Psychiatric aspects of epilepsy. In: Bhugra D, Ranjith G, Patel V. editors. Handbook of Psychiatry- A South Asian Perspective. New Delhi: Byword Viva Publishers Pvt. Ltd.; 2005. p. 117-26.

4. Mario F, Mendez A. Neuropsychiatric Aspects of Epilepsy. In: Sadock BJ and Sadock VA. Comprehensive Text Book of Psychiatry. 8th ed. Washington DC: Lippincott Williams \& Wilkins; 2005. p. 377-89.

5. Lishman WA. Epilepsy. In: Organic Psychiatry: the psychological consequences of cerebral disorder. 3rd ed. Oxford: Blackwell Science; 1997. p. 237-98.

6. Brian T. Epilepsy. In: Gelder MG, Lopez-Ibor JJ, Andreason N, eds. New Oxford Textbook of Psychiatry. New York: Oxford University Press; 2003. p. 1153-7.
7. Self-injury. http://en.wikipedia.org/wiki/Self-injurious behaviour. Online accessed at September 2010

8. American Psychiatric Association. Practice Guideline for the Assessment and Treatment of Patients with Suicidal Behaviors. Am J Psychiatry November. 2003; 160(11).

9. Hawton K, Fagg J, Marsack P. Association between epilepsy and attempted suicide. J Neurol Neurosurg Psychiatry. 1980;43:168-70.

10. World Health Organization. The world health report 1996 fighting disease, fostering development. Geneva: WHO; 1996.

11. Shakya DR. Psychiatric associations/ manifestations in seizure disorders. In: Abstract Book, BPKIHS XIV Anniversary Celebration Scientific Programme. Dharan, Nepal: BPKIHS; 2007. p. 117

12. Engel J Jr, Caldecott-Hazard S and Bandler R. Neurobiology of behavior: anatomic and physiological implications related to epilepsy. Epilepsia. 1986;27 Suppl 2:S3-13.

13. Nadkarni S, Arnedo V, Devinsky O. Psychosis in epilepsy patients. Epilepsia. 2007;48 Suppl 9:17-9. 